\section{Oral diagnosis and treatment planning: part 4. Non-carious tooth surface loss and assessment of risk}

\author{
J. A. Kaidonis ${ }^{1}$
}

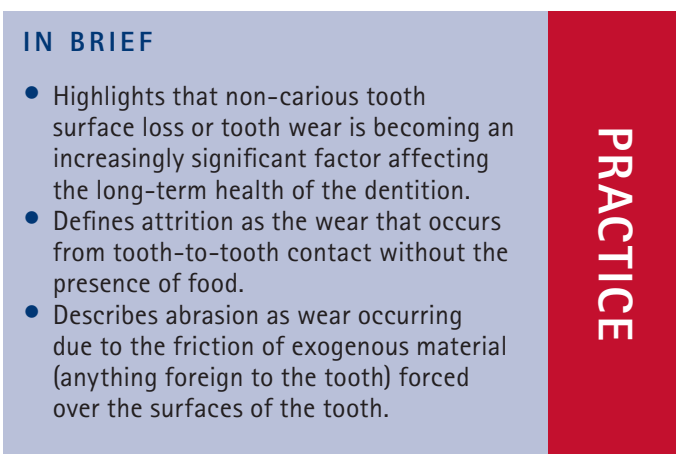

Non-carious tooth surface loss or tooth wear is becoming an increasingly significant factor affecting the long-term health
of the dentition. The adverse effects of tooth wear are becoming increasingly apparent both in young persons and, as more
people retain their teeth, into old age. This situation challenges the preventive and restorative skills of dental practitioners.

\section{INTRODUCTION}

Non-carious tooth surface loss can be diagnosed and managed following the principles of minimum intervention dentistry, where the clinician must firstly identify the clinical presence of the wear mechanism(s) in question (either attrition, abrasion, erosion or combinations thereof) and determine if the presenting mechanism(s) is active. Secondly, the clinician must identify why the patient has

\begin{tabular}{|c|c|}
\hline \multicolumn{2}{|c|}{$\begin{array}{l}\text { ORAL DIAGNOSIS } \\
\text { AND TREATMENT PLANNING* }\end{array}$} \\
\hline Part 1. & $\begin{array}{l}\text { Introduction to oral diagnosis } \\
\text { and treatment planning }\end{array}$ \\
\hline Part 2. & Dental caries and assessment of risk \\
\hline Part 3. & $\begin{array}{l}\text { Periodontal disease and assessment } \\
\text { of risk }\end{array}$ \\
\hline Part 4. & $\begin{array}{l}\text { Non-carious tooth surface loss } \\
\text { and assessment of risk }\end{array}$ \\
\hline Part 5. & $\begin{array}{l}\text { Preventive and treatment } \\
\text { planning for dental caries }\end{array}$ \\
\hline Part 6. & $\begin{array}{l}\text { Preventive and treatment } \\
\text { planning for periodontal disease }\end{array}$ \\
\hline Part 7. & $\begin{array}{l}\text { Treatment planning for } \\
\text { missing teeth }\end{array}$ \\
\hline Part 8. & $\begin{array}{l}\text { Reviews and maintenance } \\
\text { of restorations }\end{array}$ \\
\hline \multicolumn{2}{|c|}{$\begin{array}{l}\text { *This series represents chapters } 1,7,8,9,14,15,16 \text { and } 19 \text { from } \\
\text { the BDJ book A Clinical Guide to Oral Diagnosis and Treatment } \\
\text { Planning, edited by Roger Smales and Kevin Yip. All other } \\
\text { chapters are published in the complete clinical guide available } \\
\text { from the } B D J \text { Books online shop. }\end{array}$} \\
\hline
\end{tabular}

${ }^{1}$ Associate Professor, School of Dentistry, Faculty of Health Sciences, The University of Adelaide, Adelaide, South Australia 5005, Australia

Correspondence to: John Kaidonis

Email:john.kaidonis@adelaide.edu.au

Accepted 7 June 2012

DOI: 10.1038/sj.bdj.2012.722

${ }^{\circledR}$ British Dental Journal 2012; 213: 155-161 this problem and then devise a preventive and monitoring programme that is specific for the patient. Finally, where an operative approach is required, minimum intervention requiring the preservation of tooth structure is essential.

It has been well-documented that the main mechanisms of clinical tooth surface loss (TSL) or tooth wear are erosion (corrosion), attrition, and abrasion. Co-factors such as hyposalivation and hypomineralisation of the teeth may exacerbate the effects of the primary cause. As people are now retaining their teeth for longer into old age, the extent of TSL can be expected to increase further. The greatest increase in TSL has occurred from erosive wear, especially in younger persons in both dentitions. In the primary dentition, the dental pulps may be exposed. Unless it is controlled early, TSL may result subsequently in widespread exposure of dentine with hypersensitivity, an unsightly appearance of the teeth and reduced masticatory function. The costs of treatment and rehabilitation may then be considerable.

Although each individual mechanism produces TSL with clinically distinguishable characteristics, in reality the mechanisms often coexist, acting with different intensities and durations to produce clinical patterns of wear that at times are not easily identifiable. In addition to the clinical appearances that result from the above-mentioned mechanisms, characteristically different variations of TSL also exist including the wedge-shaped noncarious cervical lesion and interproximal wear. The non-carious cervical lesion is a common clinical condition that can be difficult to manage, but interproximal wear is of little consequence to the general practitioner.

The philosophy of minimum intervention dentistry has been applied successfully regarding dental caries, and the principles of minimal intervention can also be applied to the diagnosis and management of non-carious TSL. The practitioner must have sufficient background knowledge of this dental condition for its effective management. The basic clinical approach should be as follows:

- Identify the mechanism(s) causing the wear (attrition, abrasion, erosion or combinations thereof)

- Determine if the evident mechanism(s) is active

- Identify why the patient has this problem(s) and then tailor-make a preventive and monitoring programme that is specific for the patient

- Where an operative approach is required, minimum intervention requiring the preservation of tooth structure is essential.

It is important that the patient assumes responsibility for the success of the preventive treatment. The patient should be made aware of the consequences if nothing is done, both in terms of dental damage and eventual cost, versus the advantage of preventive action and its benefits. The patient then should be asked to select or choose from these alternatives, thereby making him or her take responsibility for treatment. 


\section{EROSION}

\section{Background knowledge}

Erosion (corrosion) has been defined as the chemical dissolution of tooth substance without the presence of dental plaque. In comparison to the weak plaque acids that act over relatively long periods (15-20 minutes), the acids causing erosion are moderate (approximately $\mathrm{pH}=5$ ) to strong $(\mathrm{pH}=1.2)$ acids that act on tooth surfaces over short periods (15-60 seconds). The slow percolation of plaque acids within the laminar pores of enamel rods produces subsurface demineralisation with the characteristic carious 'white spot' lesion, while those acids producing erosive demineralisation act much faster and by comparison affect only the tooth surface with very little subsurface damage.

The sources of acids that cause tooth erosion originate either from inside the body as gastric acid (intrinsic or endogenous erosion) or from outside the body as dietary, environmental or occupational acids (extrinsic or exogenous erosion). In addition to the source of the acid and its $\mathrm{pH} /$ buffering titration, the frequency of acid exposure is one of the main factors that will determine the severity or extent of the problem. Depending on the source of the acid, different erosive patterns often are observed orally, which can guide the clinician towards the correct history taking to determine the cause of the condition. This is essential if appropriate preventive measures are to be instituted. The general erosive patterns observed clinically are as follows:

- Vomiting: generally, the palatal surfaces of all the maxillary teeth are affected most because the tongue usually covers the mandibular teeth during vomiting. Immediately after the episode, gravity and the actions of the cheeks and lips during swallowing will distribute the vomitus residue to other parts of the mouth including the mandibular arch until, over time, most of the tooth surfaces are affected

- Gastro-oesophageal reflux disease (GORD): the degree of gastric reflux into the mouth varies between individuals. However, as a general rule, the refluxate rises to the back of the throat and soft palate. Usually, the palatal surfaces of the maxillary posterior teeth are affected
- Rumination, where the refluxate enters the mouth and is chewed, has been noted among bulimics and infants. Here the erosive pattern is more generalised and, in particular, includes the occlusal tooth surfaces

- Eructation (burping) where moist 'acidic air' enters the oral cavity also will have an effect. Though the palatal surfaces of the maxillary teeth are generally involved, other surfaces certainly can be affected depending whether the mouth is opened or closed during the burping action

- Dietary: as a general rule, if the clinician observes that the occlusal surfaces of the mandibular teeth are affected more than the maxillary teeth, then the agent is usually a liquid that floods the mandibular teeth. If the occlusal surfaces of both arches are equally affected, then the agent is usually solid acidic food that is masticated

- More importantly, liquids of low pH and high titratable acid also affect the palatal surfaces of the maxillary anterior and premolar teeth (Table 1). The action of swallowing causes a seal by the lips, the tightening of the cheeks up against the buccal and labial tooth surfaces, and the tongue rising hard up against the palate, thus distributing the liquid against the palatal surfaces of the maxillary teeth. Practitioners often erroneously conclude that the affected palatal surfaces of the maxillary anterior teeth are caused only by intrinsic acid

- Different actions of drinking directly from a bottle or from a glass also will produce erosion variations. Here, the labial surfaces of the anterior teeth are affected. The use of a straw usually prevents the labial surfaces of the anterior teeth being eroded. Swishing acidic drinks before swallowing may cause widespread tooth erosion involving multiple posterior tooth surfaces, while sucking citrus fruits also may affect the labial surfaces of the anterior teeth. More recently, there has been an increase in the consumption by young children of acidic sour-sweets or candies following increased targeted marketing of these products. These 'extreme' or 'intense'

\begin{tabular}{|c|c|c|c|}
\hline Item & $\mathrm{pH}$ & $\begin{array}{l}\mathrm{mmol} \mathrm{OH}^{-} / \mathrm{l} \\
\text { to } \mathrm{pH} 7.0\end{array}$ & $\begin{array}{l}\text { Erosion } \\
\text { potential }\end{array}$ \\
\hline Coca Cola & 2.6 & 34.0 & Medium \\
\hline Pepsi light & 3.1 & 34.6 & Medium \\
\hline Sprite light & 2.9 & 62.0 & High \\
\hline $\begin{array}{l}\text { Fanta } \\
\text { orange }\end{array}$ & 2.9 & 83.6 & Medium \\
\hline Red Bull & 3.4 & 91.6 & High \\
\hline $\begin{array}{l}\text { Carlsberg } \\
\text { beer }\end{array}$ & 4.4 & 40.0 & Low \\
\hline White wine & 3.7 & 70.0 & Medium \\
\hline Red wine & 3.4 & 76.6 & Medium \\
\hline Carrot juice & 4.2 & 42.0 & Low \\
\hline Apple juice & 3.4 & 82.0 & High \\
\hline $\begin{array}{l}\text { Orange } \\
\text { juice }\end{array}$ & 3.7 & 109.4 & Medium \\
\hline $\begin{array}{l}\text { Grapefruit } \\
\text { juice }\end{array}$ & 3.2 & 218.0 & High \\
\hline Milk & 7.0 & 4.0 & Low \\
\hline $\begin{array}{l}\text { Yoghurt } \\
\text { natural }\end{array}$ & 4.2 & 105.6 & Low \\
\hline $\begin{array}{l}\text { Salad } \\
\text { dressing }\end{array}$ & 3.6 & 210.0 & High \\
\hline Vinegar & 3.2 & 740.8 & High \\
\hline
\end{tabular}

sour chewable and powdered sweets may have $\mathrm{pH}$ values as low as 2.0, which is well below the critical $\mathrm{pH}$ for enamel and dentine demineralisation.

It must be stressed that long-term exposure to acids, no matter what the sources, will eventually produce a pattern where many tooth surfaces are affected, making the original source unidentifiable. In addition, missing tooth spaces, the wearing of prostheses, and individual variations of mastication and swallowing, often produce unusual characteristic patterns specific for the patient.

\section{Identifying erosion}

- The teeth lose their micro-anatomical enamel features and develop a 'glazed' or 'silky' appearance (Fig. 1)

- As the erosion progresses, teeth lose their normal contours causing curved enamel areas to flatten and to become 'dished-out' eventually. In particular, this occurs on the buccal and labial surfaces of the tooth crowns 


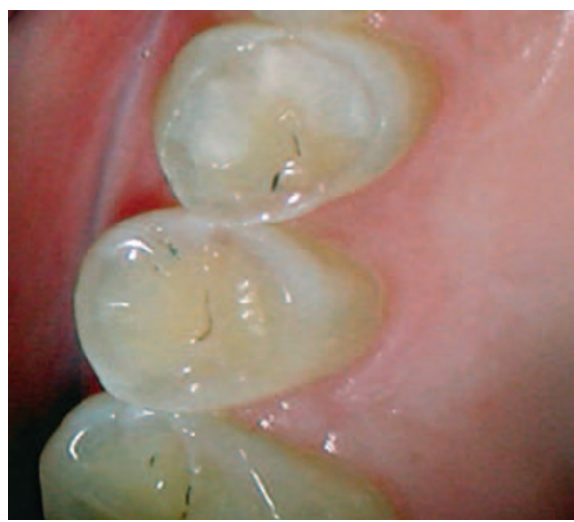

Fig. 1 Active erosion, where the teeth have lost their micro-anatomical features and appear to have a 'glazed' or 'silky' surface

- As the dentine becomes exposed, particularly on the occlusal surfaces (Fig. 2), the severity of the 'scooping' or 'cupping' increases significantly, often leaving high enamel ridges surrounding the dentine. A similar 'grooving' of the dentine occurs on the incisal edges of the anterior teeth

- The presence of non-carious cervical lesions (including wedge-shaped lesions) should also indicate to practitioners the activity of erosion (Fig. 3). Though abfraction (the flexing of teeth under load leading to noncarious cervical lesions) has been referred to in the literature as the cause of such wedge-shaped lesions, recent studies have found a multifactor explanation where toothbrush/ dentifrice abrasion and, in particular, erosion are significant aetiological co-factors.

\section{Identify activity}

- Enamel surfaces that appear 'glazed' or 'silky' demonstrate evidence of current erosive activity

\begin{tabular}{|c|c|}
\hline Score & Description \\
\hline Score 0 & $\begin{array}{l}\text { No erosive tooth wear on any } \\
\text { surfaces (excludes third molars) }\end{array}$ \\
\hline Score 1 & Initial loss of surface texture \\
\hline Score $2^{*}$ & $\begin{array}{l}\text { Distinct defect, hard tissue loss } \\
<50 \% \text { of the surface area }\end{array}$ \\
\hline Score $3^{*}$ & $\begin{array}{l}\text { Hard tissue loss }{ }^{3} 50 \% \text { of the } \\
\text { surface loss }\end{array}$ \\
\hline \multicolumn{2}{|c|}{$\begin{array}{l}\text { *Dentine often is involved but may be difficult to detect especially } \\
\text { in the cervical area. } \\
\text { The highest score found in each sextant is recorded and the six } \\
\text { scores are summed. } \\
\text { (Adapted from Bartlett et al., 2008: with permission) }\end{array}$} \\
\hline
\end{tabular}

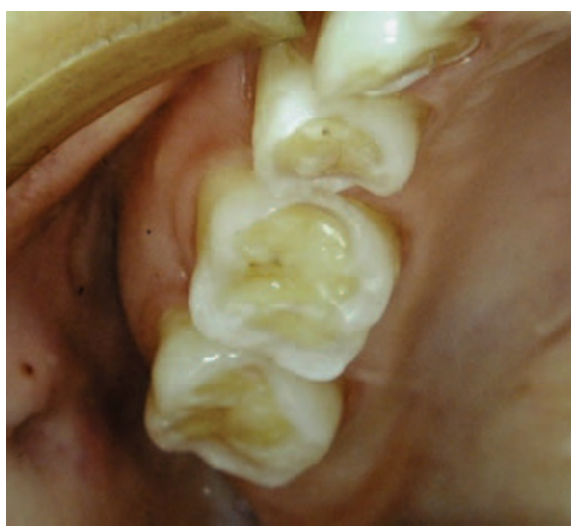

Fig. 2 Active erosion has scooped out the exposed dentine. Here, the dentinal tubules are open causing hypersensitivity

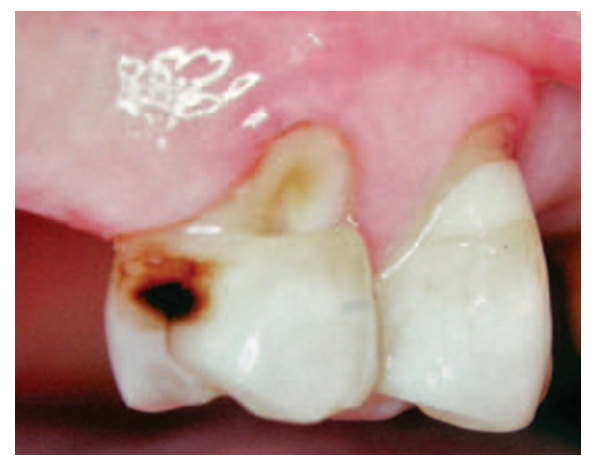

Fig. 3 A wedge-shaped non-carious cervical lesion has developed on the upper first premolar tooth. Similarly, an erosion lesion has continued to develop on the upper second premolar even after the primary lesion was restored
- Active erosion will remove the surface smear layer from exposed dentine, opening the dentinal tubules and causing dentine hypersensitivity

- If the dentition shows no evidence of plaque (especially when the mouth is in pristine condition) then this also is evidence of high erosive activity, as continuous exposure to acids will remove any evidence of biofilms in the oral cavity. Alternatively, it must be noted that in some cases erosion can be very mildly active over many years, and pristine oral conditions and dentine hypersensitivity may not be present

- Activity can also be determined using either a 'scratch test' over a short time period, or serial colour photographs (to compare changes in enamel translucency and shade from thinning of the enamel) and high-quality dental casts (to compare subtle changes in tooth morphology) over a much longer time period. The scratch test uses a no. 12 scalpel blade to score a line across an affected tooth surface, before using a small amount of a low-viscosity resin composite or an addition-cured silicone to take an impression with a wooden tongue blade, which can be labelled with the patient's name and date. Repeat the impression 1-4 weeks later and observe, using magnification, the rate of disappearance of the 'groove' in the impression

- Recently, a new basic erosive wear examination (BEWE) scoring system has been proposed for use in general dental practice where the worst-affected tooth in each quadrant is scored according to defined criteria (Table 2).

The summed score from the sextants may be used as a provisional guide to clinical management (Table 3).

\section{Identify contributing factors specific to the patient}

- The identification of the source of acid such as from dietary habits or the presence of GORD directs the clinician towards eliminating the aetiological agent(s)

\section{Table 3 Risk levels as a guide to clinical management of tooth erosion}

\begin{tabular}{l|l|l} 
Risk level & Summed score & Management \\
\hline None & $£ 2$ & $\begin{array}{l}\text { Routine maintenance and observation. Review at } \\
\text { 3 year intervals }\end{array}$ \\
\hline Low & $3-8$ & $\begin{array}{l}\text { As above plus oral hygiene, dietary assessment, advice. } \\
\text { Review at 2 year intervals }\end{array}$ \\
\hline Medium & $9-13$ & $\begin{array}{l}\text { As above plus identify main aetiological factors and } \\
\text { eliminate. Avoid restorations, apply fluorides, } \\
\text { casein-derived pastes, surface sealants. Monitor at } \\
6-12 \text { month intervals (casts, photos, scratch tests) }\end{array}$ \\
\hline High & ${ }^{3} 14$ & $\begin{array}{l}\text { As above plus minimal restorations } \\
\text { (Adapted from Bartlett et al., 2008: with permission) }\end{array}$ \\
\hline
\end{tabular}


- A reduced quality and quantity of saliva are important contributing factors. Xerostomia (the sensation of dry mouth) or varying degrees of hyposalivation, either diurnally or nocturnally, are definite risk factors that may be responsible for erosion even with the mildest exposure to acid. For example, a dietary history or a dietary analysis showing what seem to be insignificant exposures to acids should never be ignored. In particular, medications that cause hyposalivation need to be identified, and appropriate tests should be considered for assessing and monitoring the quality and quantity of the saliva

- The age of the patient also should be considered. Newly erupted teeth have enamel crystals containing high amounts of carbonated hydroxyapatite that will dissolve more readily in acids than 'matured' enamel, which has a higher percentage of fluorapatite and other minerals

- Past and present fluoride experience must be considered as another risk factor.

Prevention (should be specific for that patient):

- The most important single factor that the clinician must focus on is to identify and eliminate the acidic aetiological agent(s) whether it is a dietary soft drink, a wine or a commonly consumed food such as pickled vegetables. Often, the pattern of erosion observed within the oral cavity will direct the clinician's history taking to an identifiable cause. GORD may require confirmation/intervention by referral to a medical practitioner

- Rinsing with water during known times of acid exposure, such as immediately after a bulimic episode, is very effective in eliminating erosive damage

- Various remineralising products such as fluorides and casein-derived pastes containing Recaldent (CPP-ACP: casein phosphopeptide-amorphous calcium phosphate) are very useful. Recaldent contains all the raw products for remineralisation to occur and is particularly effective when the $\mathrm{pH}$ drops, causing the protein (CPP) to release the calcium and phosphate ions at the time of need.

Though opinions vary on the dose requirements for such products, as a general rule low-dose 'continuous' fluoride exposure can be effective provided sufficient calcium and phosphate ions are available in the saliva. For example, a low-dose fluoride mouth rinse used three times daily, or the regular placement of a small 'bead' of fluoridated toothpaste on the tongue and spreading it throughout the mouth without rinsing are useful regimes. However, there are times when $1.23 \%$ acidulated phosphate fluoride is more effective as the ions required for remineralisation are derived from the tooth surface. Similarly, products such as stannous fluoride and other fluorides are effective because they can precipitate on the tooth surface to form a protective barrier to acids. The strategic use of Recaldent products is also important, such as GC Tooth Mousse Crème/PROSPECT MI Paste, and Tooth Mousse Plus/PROSPECT MI Plus (with fluoride). The CPP-ACP complexes also readily incorporate fluoride ions to form CPP-ACFP. These products may be smeared over the teeth just before bedtime in a patient who has hyposalivation and nocturnal gastric reflux. The Recaldent products act as an artificial pellicle allowing the calcium and phosphate ions to be released as soon as the $\mathrm{pH}$ drops.

\section{ATTRITION}

\section{Background knowledge}

Attrition is the wear that occurs from tooth-to-tooth contact without the presence of food. It occurs from tooth grinding either nocturnally while asleep or diurnally. Although researchers have over the years reported that occlusal interferences and sleep disturbances are possible causes, it is commonly accepted that either chronic 'stress' or 'distress' seem to be the main risk factors. 'Highly-strung' individuals and those who chronically worry have the propensity to grind their teeth.

The grinding action follows a pattern where commonly the grinding stroke is from centric occlusion (maximum intercuspal position) to eccentric lateral positions (Fig. 4). The tooth contact movement is past the canine edge-to-edge position into an extreme lateral position. In severe cases

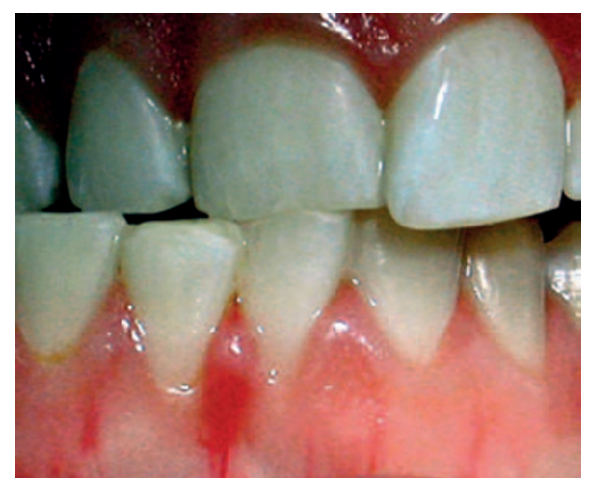

Fig. 4 The mandible is positioned in an extreme eccentric movement. This common grinding position reflects the load imposed on the upper right central incisor, where the tooth is worn incisally and whose facet fits perfectly with those on the lower anterior teeth

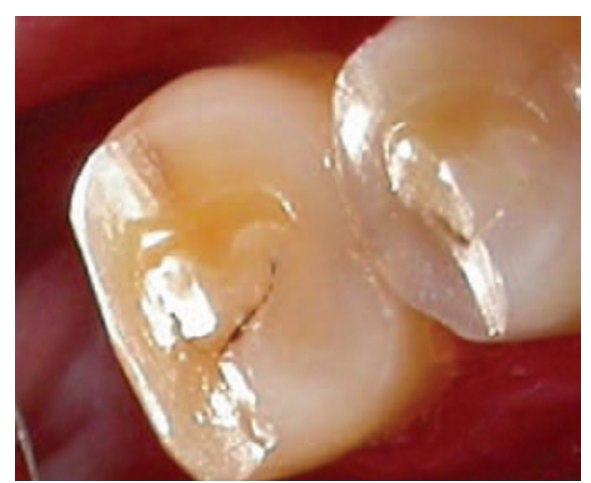

Fig. 5 The occlusal surfaces of these upper premolars show distinct shiny facets that reflect active tooth grinding. Note the buccopalatal directed striations indicating the lateral mandibular movement that caused them

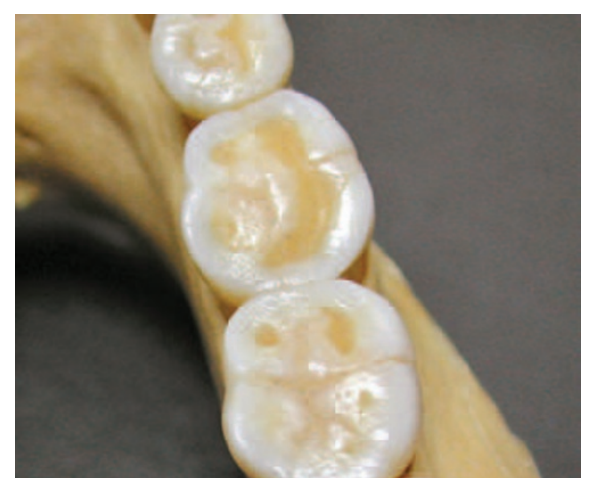

Fig. 6 Skeletal material shows evidence of abrasion on the occlusal surfaces of the molar teeth. Note the scooped dentine and the gouged and scratched enamel reflecting the diet of the individual. The first molar tooth has been in the mouth for a longer time and, thus, is more worn than the second molar

this action can cause pathological tooth damage such as enamel flaking and cusp fracture. The enamel flaking is observed generally on the labial incisal edges of the maxillary and the lingual incisal edges of the mandibular anterior teeth. Similarly, the buccal cusps of the maxillary and the 
lingual cusps of the mandibular posterior teeth predominantly fracture.

The common feature seen on teeth from grinding is the facet, described as a relatively flat area with a well-circumscribed border (Fig. 5). If attrition is the only or most predominantly active mechanism occurring, any exposed dentine remains flat and shows no evidence of scooping as is observed with abrasion and erosion. In addition, facets seen clinically on the teeth will have a matching facet on the teeth in the opposing arch. Facets with less well-defined borders or with evidence of dentinal scooping reflect the presence of superimposed abrasion and/or erosion.

Reports on the prevalence of tooth grinding vary considerably in the literature, where figures from 5-90\% have been reported, depending on the research methods applied. Where subjective questionnaires have been used, the levels are usually very low. However, where facet frequencies have been objectively quantified within populations, the prevalence is high. Although opinions vary, at such levels the tooth grinding behaviour can be considered as physiologic, where everyone tooth grinds to some degree depending on life events. However, if the behaviour becomes frequent and excessive, and the stomatognathic system cannot adapt or is too slow to adapt, then certain craniomandibular disorders may result. In addition, the wear rate may increase to become excessive for a person's age, leading to pathological tooth wear, so that the teeth will not last the life of the individual, hence requiring intervention. Finally, tooth grinding behaviour also has been reported in mentally-retarded and comatose patients, an indication that the 'grinding centre' is in the 'primitive' part of the brain.

\section{Identifying attrition}

- Presence of facets

- Enamel flaking and cusp fractures

- Symptoms of various craniomandibular disorders.

\section{Identify activity}

- Can be determined using the scratch test described previously

- Well-demarcated tooth facets are present, even when other TSL mechanisms coexist

- Shiny facets will reflect light at the appropriate angulations of the dental mirror and operator light (indicates

high activity)

- Flaked enamel on the incisor teeth is not stained (implies that the flaking has happened recently, therefore the tooth grinding is active)

- Patient has been aware of tooth grinding (active)

- Symptoms of myofascial pain dysfunction with associated limitation of jaw opening (active).

\section{Identify contributing factors specific to the patient}

- Chronic stress, whether it is related to lifestyle, personality trait, or various mental disorders, appears to be the common denominator for active tooth grinding

- Current opinion as to the effects of the occlusion on tooth grinding does not support an association between tooth grinding and occlusal interferences. Not that occlusion is unimportant. Occlusal interferences that have developed slowly over time during growth and development generally allow the stomatognathic system to adapt to its own functional cycle. However, iatrogenic occlusal interferences that are suddenly introduced into the system become obstacles to an already developed pattern. The lack of time to adapt often causes problems. A history of recent dental treatment is relevant to some craniomandibular disorders

- Some craniofacial types are predisposed to certain patterns of occlusal wear. Angle Class II division 2 malocclusions with deep anterior overbites tend to result in excessive anterior tooth wear, while the posterior teeth remain relatively unaffected. Children in particular with such malocclusions would gain long-term benefits from orthodontic treatment.

Prevention (should be specific for that patient):

- Acrylic resin nightguard/splint (this will prevent opposing tooth contacts and hence reduce their rate of wear)

- Stress management or appropriate referral for psychological assessment may at times be appropriate

- In vitro studies using GC Tooth
Mousse Crème as a lubricant over occlusal enamel and dentine surfaces during simulated tooth grinding have been shown to reduce occlusal wear significantly.

\section{ABRASION}

\section{Background knowledge}

Abrasion is the wear that occurs by the friction of exogenous material (anything foreign to the tooth) that is forced over the surfaces of the tooth. The most common form of abrasion, and overlooked by most dentists, is that produced by food. Though food abrasion was very evident in pre-contemporary hunter-gatherer populations, it is not usually a problem in current populations because of the high degree of food processing and hence the consumption of relatively soft foods. It has been stated that the prolonged retention of tooth cusps throughout life in the human dentition is a relatively recent occurrence from an evolutionary perspective, and that although past human dentitions were excessively worn, by abrasive diets, they maintained masticatory efficiency through the differential wear between enamel and dentine. In our modern societies, although abrasion from food is comparatively minimal, it often coexists with erosion, which produces an increased wear rate. This wear is often called 'erosive tooth wear'

The wear from food abrasion is generally distributed throughout the arch and, when dentine is exposed, it is scooped out because it is softer than enamel. Interestingly, such exposures show no sensitivity because the dentinal tubules are covered by a smear layer resulting from mechanical friction between the teeth and food. This is not dissimilar to the frictional smear layer produced by rotary instruments.

At times, certain types of habitually eaten hard foods such as nuts and seeds may cause abrasion on specific tooth surfaces. Occupational habits also may cause abrasive wear on specific teeth, such as holding hairdresser bobby pins or hairpins between the incisors. Dusty working environments, which occur in iron-works, mines and quarries, may lead to increased tooth abrasion. Microwear detail of non-carious cervical lesions show evidence of abrasive wear from the toothbrush/ dentifrice used (though 
evidence of superimposed erosion also has been reported). Using interdental wooden toothsticks may cause abrasive wear of the adjacent proximal root surfaces.

\section{Identifying abrasion}

- Abrasion from foreign objects such as tobacco pipe stems and bobby hairpins, or from hard foods such as watermelon and pumpkin seeds (passively nibbled incisally on the anterior teeth), is often identified by asymmetric wear in the form of a 'notch' on the anterior teeth

- Scooped dentine on the incisal and occlusal surfaces (Fig. 6), especially if the exposed dentine is not sensitive, may be caused by abrasion - because we have to eat. However, in our modern culture, food is not generally abrasive and often there is superimposed tooth erosion, which makes clinical identification of the cause difficult

- Although concepts of abfraction should not be discounted, a wedgeshaped non-carious cervical lesion is a strong indication that toothbrush/ dentifrice abrasion is an aetiological factor though, again, superimposed erosion may be a significant contributor. Usually, the presence of active superimposed erosion will exhibit some degree of sensitivity in the affected cervical areas.

\section{Identify activity}

- Identifying abrasion is difficult because of the common contributing effect of erosion. It may be argued that identifying occlusal abrasion may not be necessary because of the widespread consumption of soft refined foods

- As for abrasion caused by foreign objects, a history taken showing a continuing habit, such as the continual use of a pipe, is sufficient to indicate activity

- Serial colour photographs or dental casts can show a progression in wear.

\section{Identify contributing factors specific to the patient}

- A history showing the frequent consumption of abrasive foods such as nuts and seeds

- Erosion may be a contributing factor. Acids that 'soften' tooth surfaces may accentuate the TSL effects of mechanical actions that cause abrasion and, indeed, attrition

- Newly erupted teeth, primary teeth in particular, that have not 'matured' may be more prone to abrasion

- Excessively loaded anterior teeth will wear faster when there are edentulous posterior segments from previous extractions.

Prevention (should be tailor-made specific for that patient):

- Preventing abrasion requires the taking of a careful history to identify abrasive dentifrices, foods, foreign objects, or common habits that are causing the problem

- Making the patient aware of the problem and taking responsibility for the prevention of further tooth wear can have a profoundly positive effect

- Operative dentistry to restore a full complement of occluding teeth will allow a better distribution of load and hence a reduction of occlusal abrasion.

\section{RESTORATION WEAR}

Restorative materials are subject to the same wear processes as tooth structure, but with far more variation in their susceptibility. The wear processes in materials are usually described using scientific rather than clinical dental terminology.

Most in vitro studies of restorative materials have focused on abrasive wear, either from toothbrushing/dentifrices or from mastication. Many in vitro studies also have examined the antagonistic wear of teeth and restorative materials, because excessive differential clinical wear between them may lead potentially to adverse biological, functional and aesthetic effects. The lowest antagonistic wear rates appear to occur with amalgam and cast metal alloys, microfilled and nanofilled resin composites, and machined ceramics. Severe tooth damage may occur from rough ceramic material surfaces.

Few in vitro studies have examined acid erosive wear of restorative materials. Conventional glass-ionomer cements and resin-modified glass-ionomer cements are more susceptible clinically to abrasive and erosive wear than other commonly used restorative materials. Severe hyposalivation may exacerbate the effects of erosion.
The surfaces of ceramic and resin composite materials that contain glass particles are susceptible to damage from acidulated fluoride gels.

\section{ASSESSMENT OF RISK TO TOOTH SURFACE LOSS}

The process of active TSL is most severe when several primary causes and several co-factors such as hyposalivation, abrasive restorative materials, defective tooth structure and lack of posterior tooth support are present. The extent and rate of TSL will be influenced by the severity of tooth grinding (attrition), sources of dietary and gastric acids (erosion), and incorrect toothbrushing/dentifrices (abrasion). The patient's occupation, medical and dental histories and lifestyle should be assessed for possible risk factors. In some instances, there may be medico-legal issues to be aware of. The basic erosive wear examination (BEWE) index provides some clinical guidance for the risk assessment and management of tooth erosion.

\section{MONITORING TOOTH WEAR}

Monitoring is an important part of minimal intervention dentistry and should be considered as routine. Once a preventive approach or any other form of treatment is implemented, follow-up assessments should be made to see if the measures undertaken are successful. Monitoring requires a complete reassessment where the history, special tests, colour photographs and dental casts are repeated, to note either improvement or otherwise from the original assessment. For example, if a subsequent scratch test indicates no TSL activity when compared to the initial test, or if previous tooth sensitivity is no longer occurring, then this is an indication that the measures adopted are working.

\section{FURTHER READING}

Bartlett D, Ganss C, Lussi A. Basic erosive wear examination (BEWE): a new scoring system for scientific and clinical needs. Clin Oral Investig 2008 : 12(Suppl 1): S65-S68.

Berry D C, Poole D F. Attrition: possible mechanisms of compensation. J Oral Rehabil 1976; 3: 201-206. Edgar M, O'Mullane D, Dawes C (eds). Saliva and oral health. 3rd ed. London: British Dental Association, 2004. Grippo J 0, Simring M, Schreiner S. Attrition, abrasion, corrosion and abfraction revisited: a new perspective on tooth surface lesions. J Am Dent Assoc 2004; 135: 1109-1118. (Erratum: 2004; 135: 1376).

Hunter M L, Addy M, Pickles M J, Joiner A. The role of toothpastes and toothbrushes in the aetiology of tooth wear. Int Dent J 2002; 52: 399-405. 
Imfeld T. Dental erosion. Definition, classification and links. Eur J Oral Sci 1996; 104: 151-155.

Jaeggi T, Lussi A. Prevalence, incidence and distribution of erosion. In Lussi A (ed) Dental erosion. From diagnosis to therapy. Monogr Oral Sci 2006. 20: 44-65.

Kaidonis J A. Tooth wear: the view of the anthropologist. Clin Oral Investig 2008; 12(Suppl 1): S21-S26 (Erratum: S69).

Kaidonis J A, Richards L C, Townsend G C. Non-carious changes to tooth crowns. In Mount G J, Hume W R (eds). Preservation and restoration of tooth structure. 2 nd ed. pp 47-60. Brisbane: Knowledge Books and Software, 2005.

Loewen R R, Marolt R J, Ruby J D. Pucker up - the effects of sour candy on your patient's oral health. A review of the dental erosion literature and $\mathrm{pH}$ values for popular candies. Northwest Dent 2008; 87: 20-33.
Lussi A, Jaeggi T. Chemical factors. In Lussi A (ed) Dental erosion. From diagnosis to therapy. Monogr Oral Sci 2006. 20: 77-87.

Lussi A, Jaeggi T, Zero D. The role of diet in the aetiology of dental erosion. Caries Res 2004; 38: 34-44.

Mair L H, Stolarski T A, Vowles R W, Lloyd C H. Wear: mechanisms, manifestations and measurement. Report of a workshop. J Dent 1996; 24: 141-148.

Nguyen C, Ranjitkar S, Kaidonis J A, Townend G C. A qualitative assessment of non-carious cervical lesions in extracted human teeth. Aust Dent J 2008; 53: 46-51.

Ranjitkar S, Narayana T, Kaidonis J A, Hughes T E, Richards L C, Townsend G C. The effect of casein phosphopeptide -amorphous calcium phosphate on erosive dentine wear. Aust Dent J 2009; 54: 101-107.
Reynolds E C. Anticariogenic complexes of amorphous calcium phosphate stabilized by casein phosphopeptides: a review. Spec Care Dent 1998; 18: 8-16.

Shabanian M, Richards L C. In vitro wear rates of materials under different loads and varying $\mathrm{pH}$. J Prosthet Dent 2002; 87: 650-656. (Erratum: 2002; 88: 554).

Tyas M J, Anusavice K J, Frencken J E, Mount G J. Minimal intervention dentistry - a review. FDI Commission Project 1-97. Int Dent J 2000; 50: 1-12. Yip K H, Peng D, Smales R J. Effects of APF gel on the physical structure of compomers and glass ionomer cements. Oper Dent 2001; 26: 231-238.

Yip K H, Smales R J, Kaidonis J A. Tooth erosion: prevention and treatment. New Delhi: Jaypee Brothers Medical Publishers, 2006. 